\title{
QUASICONFORMAL MAPPINGS AND ROYDEN ALGEBRAS IN SPACE $\left({ }^{1}\right)$
}

\author{
BY \\ LAWRENCE G. LEWIS
}

\begin{abstract}
On every open connected set $G$ in Euclidean $n$-space $R^{n}$ and for every index $p>1$, we define the Royden $p$-algebra $M_{p}(G)$. We use results by F. W. Gehring and W. P. Ziemer to prove that two such sets $G$ and $G^{\prime}$ are quasiconformally equivalent if and only if their Royden $n$-algebras are isomorphic as Banach algebras. Moreover, every such algebra isomorphism is given by composition with a quasiconformal homeomorphism between $G$ and $G^{\prime}$. This generalizes a theorem by $\mathbf{M}$. Nakai concerning Riemann surfaces. In case $p \neq n$, the only homeomorphisms which induce an isomorphism of the $p$-algebras are the locally bi-Lipschitz mappings, and for $1<p<n$, every such isomorphism arises this way. Under certain restrictions on the domains, these results extend to the Sobolev space $H_{p}^{1}(G)$ and characterize those homeomorphisms which preserve the $H_{p}^{1}$ classes.
\end{abstract}

Introduction. In 1960 Nakai proved [12] that two Riemann surfaces are quasiconformally equivalent if and only if their Royden algebras are isomorphic. In this paper we characterize a class of homeomorphisms and a family of Banach algebras which extend this result to higher dimensions.

On each finite subset $G$ of Euclidean $n$-space $R^{n}$ we define the Royden $p$-algebra $M_{p}(G)$ for arbitrary $p>1 . M_{p}(G)$ is a commutative semisimple Banach algebra with identity. We then form the Gelfand compactification of $G$ with respect to the Royden $p$-algebra and call it the Royden $p$-compactification of $G$ [15].

We also define $Q_{p}$-mappings of $G$ onto $G^{\prime}$ and characterize them to be exactly those homeomorphisms which induce by composition an isomorphism between the respective Royden $p$-algebras. Using the Gelfand theory we prove that for $1<p \leqq n$ every such isomorphism is obtained by composition with a $Q_{p}$-mapping.

If $G$ has finite measure we may consider $M_{p}(G)$ to be a subset of the Sobolev space $H_{p}^{1}(G)$. With certain restrictions on the domains, we also prove that the $Q_{p^{-}}$ mappings are exactly those homeomorphisms which leave the $H_{p}^{1}$ classes invariant.

Recently F. W. Gehring has shown that all $Q_{p}$-mappings are quasiconformal mappings. In fact for every $p \neq n$ they are precisely the class of all bi-Lipschitz

Presented to the Society, January 23, 1969; received by the editors December 2, 1969 and, in revised form, March 9, 1970.

AMS 1970 subject classifications. Primary 30A60, 46J15; Secondary 46E35, 31B15.

Key words and phrases. Royden algebra, quasiconformal mappings, Sobolev space, Royden compactification, bi-Lipschitz mapping, $p$-capacity, conformal capacity.

${ }^{(1)}$ This paper is based on the author's dissertation at Indiana University, May, 1969. The research was partially supported by NSF Grant Nos. GP-4071 and GP-7505. 
mappings. Thus the case $p=n$ is distinguished when studying either the Royden algebras or the Sobolev spaces under composition. This special case was introduced by C. Loewner [8] as "conformal capacity" and studied by F. W. Gehring [4].

H. M. Reimann [14] has recently considered a class of homeomorphisms similar to the $Q_{p}$-mappings.

The author wishes to express appreciation to Professor George Springer for his inspiration as thesis advisor and to Professors Frederick Gehring and William Ziemer for their many helpful suggestions.

1. Preliminaries. The following notation is used: $m_{n}$ is $n$-dimensional Lebesgue measure, $m_{n-1}$ is $(n-1)$-dimensional Hausdorff measure, $L_{p}(G)$ is the usual space of equivalence classes of functions $f$ for which $|f|^{p}$ is $m_{n}$-integrable, with norm $\|f\|_{p}$, and $C_{0}^{\infty}(G)$ is the space of infinitely differentiable functions with compact support contained in $G$. Unless otherwise indicated, all functions are complexvalued and all integrals are taken over $G$ (or $\left.G^{\prime}\right) . x=\left(x^{1}, \ldots, x^{n}\right)$ denotes an arbitrary point in $R^{n}, B(x, r)$ and $S(x, r)$ are the usual ball and sphere, abbreviated $B^{n}$ and $S^{n-1}$ in case $x=0$ and $r=1$, and $\omega_{n}=m_{n-1}\left(S^{n-1}\right)$.

Denote by $\operatorname{ACL}(G)$ the collection of all functions which are absolutely continuous along the intersection of every compact $n$-interval with $m_{n-1}$-almost every line parallel to the coordinate axes. Note that every $f \in \operatorname{ACL}(G)$ has a gradient.

More generally, a function $f$ has a (weak) gradient

$$
\nabla f=\left(r^{1}, \ldots, r^{n}\right)
$$

if there exist distributional derivatives $r^{i}$ satisfying

$$
\int w r^{i} d m_{n}=-\int f \frac{\partial w}{\partial x^{i}} d m_{n}, \quad w \in C_{0}^{\infty}(G),
$$

for $i=1, \ldots, n$. Then the Dirichlet p-integral of $f$ is

$$
D_{p}[f]=\int|\nabla f|^{p} d m_{n}=\int\left[\sum_{i=1}^{n}\left|r^{i}\right|^{2}\right]^{p / 2} d m_{n} .
$$

In case $f$ has locally integrable partial derivatives, they are equal $m_{n}$-a.e. to the $r^{1}$, and hence satisfy (1.1). Thus, for $f \in \operatorname{ACL}(G)$ with $D_{p}[f]<\infty, \nabla f$ as defined above is the usual gradient.

A real-valued function $u$ is of class $H_{p}^{1}$ on $G$ if $u \in L_{p}(G)$ and if $u$ has a gradient and satisfies $D_{p}[u]<\infty$. A homeomorphism is of class $H_{p}^{1}$ on $G$ if each of its coordinate functions is. $H_{p}^{1}(G)$ is the space of all such functions with the norm

$$
\|u\|=\left\{\int\left[|u|^{2}+|\nabla u|^{2}\right]^{p / 2} d m_{n}\right\}^{1 / p} .
$$

As is customary with $L_{p}(G)$ we write $u \in H_{p}^{1}(G)$ and mean that $u$ is of class $H_{p}^{1}$ on G. $H_{p}^{1}(G)$ is complete in this norm, and each of its equivalence classes contains a 
representative in $\operatorname{ACL}(G)[10$, p. 66]. This fact together with Fubini's theorem implies the following.

LEMMA 1.1. A continuous real-valued function $u$ which is locally of class $H_{p}^{1}$ on $G$ is in $\operatorname{ACL}(G)$.

A ring $R$ is a connected open set in $R^{n}$ whose complement consists of a bounded component $C_{0}$ and an unbounded one $C_{1}$. For $1<p<\infty$, the p-capacity of $R$ is

$$
\operatorname{cap}_{p} R=\inf \left\{\int_{R}|\nabla u|^{p} d m_{n}\right\}
$$

where the infimum is taken over all continuous functions $u \in \operatorname{ACL}(R)$ with constant boundary values 0 and 1 . As in [3, p. 358] each such function may be extended to $R^{n}$ and the integral taken over $R^{n}$ without affecting cap ${ }_{p}$. Such an extended function is admissible for $R$.

In case $R$ is the spherical ring $\left\{x: a<\left|x-x_{0}\right|<b\right\}$, then

$$
\begin{aligned}
& \operatorname{cap}_{p} R=\omega_{n}\left\{\int_{a}^{b} t^{q-1} d t\right\}^{1-p}=\omega_{n}(\log b / a)^{1-n}, \quad p=n, \\
& =\omega_{n}\left(\left(b^{q}-a^{q}\right) / q\right)^{1-p}, \quad p \neq n,
\end{aligned}
$$

where $q=(p-n) /(p-1)$. Note that this gives easily the existence of a unique extremal function for $\operatorname{cap}_{p} R$ whenever $R$ is a spherical ring; for $p=n$ this is well known and for $p \neq n$ it is the function $|x|^{q}$ with proper normalization. The uniqueness follows from Clarkson's inequality as in [11, pp. 74, 77].

2. The Royden $p$-algebra. Denote by $M_{p}(G)$ the collection of all continuous functions $f$ such that

(i) $f \in \operatorname{ACL}(G)$,

(ii) $\|f\|_{\infty}=\sup _{G}|f|<\infty$,

(iii) $D_{p}[f]<\infty$.

Since Minkowski's inequality implies

$$
D_{p}[f g]^{1 / p} \leqq\|f\|_{\infty} D_{p}[g]^{1 / p}+\|g\|_{\infty} D_{p}[f]^{1 / p},
$$

it follows that $M_{p}(G)$ is an algebra under pointwise multiplication with the constant function 1 as identity.

THEOREM 2.1. With the norm

$$
\|f\|=\|f\|_{\infty}+D_{p}[f]^{1 / p},
$$

$M_{p}(G)$ is a complex commutative Banach algebra with identity.

Proof. We conclude from (2.1) that $\|f g\| \leqq\|f\|\|g\|$, and since $\|1\|=1$ we only need to show that $M_{p}(G)$ is complete in the norm (2.2) to finish the proof.

Let $\left\{f_{j}\right\}$ be a Cauchy sequence in $M_{p}(G)$. Then $\left\{f_{j}\right\}$ converges uniformly on $G$ to a bounded continuous function $f$, and the derivatives $\left\{r_{j}^{i}\right\}$ form a Cauchy sequence in 
$L_{p}(G), i=1, \ldots, n$. There exist functions $r^{1}, \ldots, r^{n} \in L_{p}(G)$ such that $\left\|r_{j}^{i}-r^{i}\right\|_{p} \rightarrow 0$, and an easy calculation shows that $f$ and $r^{i}$ satisfy (1.1). Thus $f$ has a gradient satisfying $D_{p}[f]<\infty$, and $f$ is locally of class $H_{p}^{1}$ on $G$. Finally Lemma 1.1 implies that $f \in M_{p}(G)$, proving the theorem.

A sequence $\left\{f_{j}\right\}$ of functions in $M_{p}(G)$ converges in the $\mathrm{BD}_{p}$-topology to $f$ if

(i) $\left\|f_{j}-f\right\|_{\infty} \leqq M<\infty$,

(ii) $f_{j} \rightarrow f$ uniformly on compact subsets of $G$,

(iii) $D_{p}\left[f_{j}-f\right] \rightarrow 0$.

COROLlaRY 2.2. $M_{p}(G)$ is complete in the $\mathrm{BD}_{p}$-topology.

Proof. Any Cauchy sequence in the $\mathrm{BD}_{p}$-topology converges uniformly on compact subsets to a bounded continuous function, and the proof is similar to the above proof.

3. Induced isomorphisms. Let $T$ be a homeomorphism of $G$ onto $G^{\prime}$. Denote $T \in Q_{p}(K)=Q_{p}(K ; G)$ if for some $K<\infty$

$$
\operatorname{cap}_{p} T(R) \leqq K \operatorname{cap}_{p} R
$$

for every spherical ring $R$ satisfying $\bar{R} \subset G$. Tis called a $Q_{p}$-mapping if $T \in Q_{p}(K ; G)$ and $T^{-1} \in Q_{p}\left(K ; G^{\prime}\right)$ for some $K . T$ is a quasiconformal mapping if for some $1 \leqq K<\infty$

$$
K^{-1} \operatorname{cap}_{n} R \leqq \operatorname{cap}_{n} T(R) \leqq K \operatorname{cap}_{n} R
$$

for every ring $R$ satisfying $\bar{R} \subset G$.

The restriction to spherical rings in the definition of $Q_{p}(K)$ is necessary for the additivity of the $p$-capacity in (4.3).

RemarK 3.1. It is well known that a $Q_{n}$-mapping is a quasiconformal mapping (see [3], [11] or [19]). Thus every $Q_{p}$-mapping is a quasiconformal mapping [5, Theorem 1] and is in fact a bi-Lipschitz mapping for $p \neq n$ [5, Theorem 2]. It follows easily (see Corollary 3.3) that the class of $Q_{p}$-mappings for all $p \neq n$ is precisely the class of all homeomorphisms $T$ which together with their inverses satisfy the following Lipschitz condition: There exists an $M<\infty$ such that

$$
\limsup _{|x-y| \rightarrow 0} \frac{|T(x)-T(y)|}{|x-y|} \leqq M, \quad x \in G .
$$

THEOREM 3.2. Every $Q_{p}$-mapping $T$ of $G$ onto $G^{\prime}$ induces an algebra isomorphism $\varphi_{T}$ of $M_{p}\left(G^{\prime}\right)$ onto $M_{p}(G)$ defined by

$$
\varphi_{T}(f)=f \circ T, \quad f \in M_{p}\left(G^{\prime}\right) .
$$

Proof. Since the composition $\varphi_{T}$ clearly preserves the algebraic operations, we prove only $\varphi_{T}\left(M_{p}\left(G^{\prime}\right)\right) \subset M_{p}(G)$. This will complete the proof since the opposite inclusion follows similarly. 
Let $f \in M_{p}\left(G^{\prime}\right)$ and set $u=\operatorname{Re} f$ and $v=u \circ T$. In case $p \neq n$ it follows from (3.1) and the Rademacher-Stepanoff theorem [17, p. 310] that $T$ and $T^{-1}$ are differentiable $m_{n}$-a.e. and that

$$
M^{-n} \leqq|J T| \leqq M^{n} \quad m_{n} \text {-a.e. in } G,
$$

for some $M<\infty$. Again using (3.1), we get

$$
|\nabla v| \leqq M|\nabla u| \circ T \quad m_{n} \text {-a.e. in } G \text {. }
$$

Then (3.3) and (3.4) yield

$$
\begin{aligned}
\int_{G}|\nabla v|^{p} d m_{n} & \leqq M^{p} \int_{G}|\nabla u|^{p} \circ T d m_{n} \\
& \leqq M^{p+n} \int_{G}|\nabla u|^{p} \circ T|J T| d m_{n}=M^{p+n} \int_{G}|\nabla u|^{p} d m_{n} .
\end{aligned}
$$

For $p=n$ let $Q$ be any $n$-cube satisfying $\bar{Q} \subset G$ and denote $Q^{\prime}=T(Q), u_{0}=u \mid Q^{\prime}$ and $v_{0}=u_{0} \circ T \mid Q$. Then $u_{0} \in H_{n}^{1}\left(Q^{\prime}\right)$ and it follows from [20, Remark 4.2] that $v_{0} \in H_{1}^{1}(Q)$ and

$$
\int_{Q}\left|\nabla v_{0}\right|^{n} d m_{n} \leqq K \int_{Q^{\prime}}\left|\nabla u_{0}\right|^{n} d m_{n}
$$

Exhausting $G$ by a sequence of disjoint cubes gives

$$
\int_{G}|\nabla v|^{n} d m_{n} \leqq K \int_{G^{\prime}}|\nabla u|^{n} d m_{n}
$$

We apply the above argument to $\operatorname{Im} f$ and see that for any $1<p<\infty$ there exists a constant $K_{2}$ depending only on $K, M, p$ and $n$ such that

$$
D_{p}[g] \leqq K_{2} D_{p}[f],
$$

where $g=f \circ T, f \in M_{p}\left(G^{\prime}\right)$. Lemma 1.1 then implies that $g \in M_{p}(G)$, concluding the proof.

COROLlaRY 3.3. Every homeomorphism $T$ of $G$ onto $G^{\prime}$ such that $T$ and $T^{-1}$ satisfy (3.1) is a $Q_{p}$-mapping for all $p$.

Proof. Such a $T$ satisfies (3.3) and (3.4). Let $R^{\prime}$ be a spherical ring in $G^{\prime}, \bar{R} \subset G^{\prime}$, and let $u$ be the extremal function for $\operatorname{cap}_{p} R^{\prime}$. Setting $v=u \circ T$, the above proof gives

$$
\operatorname{cap}_{p} R \leqq \int_{G}|\nabla v|^{p} d m_{n} \leqq M^{p+n} \int_{G^{\prime}}|\nabla u|^{p} d m_{n}=M^{p+n} \operatorname{cap}_{p} R^{\prime}
$$

where $R=T^{-1}\left(R^{\prime}\right)$. Hence $T^{-1} \in Q_{p}\left(M^{p+n}\right)$ and the corollary follows by applying the same argument to $T$.

4. Characterization of $Q_{p}$-mappings. In this section we give a necessary and sufficient condition for a given homeomorphism $T$ of $G$ onto $G^{\prime}$ to be a $Q_{p}$-mapping. 
Suppose that $T$ has the property that $u=v \circ T^{-1}$ is admissible for $T(R)$ whenever $v$ is the extremal function for $\operatorname{cap}_{p} R$ for any spherical ring satisfying $\vec{R} \subset G$; i.e., suppose $T^{-1}$ preserves ACL functions. Suppose further that there exists a constant $K_{1}<\infty$ independent of $R$ such that

$$
D_{p}[u]^{1 / p} \leqq K_{1}\|v\|
$$

whenever $u$ and $v$ are as above.

LEMMA 4.1. $T \in Q_{p}(K)$ for some $K \leqq K_{1}^{p}$.

Proof. If the lemma were false there would exist a spherical ring $R=$ $\left\{x: a<\left|x-x_{0}\right|<b\right\}$ with $\bar{R} \subset G$ such that

$$
\operatorname{cap}_{p} T(R) \geqq d^{p} \operatorname{cap}_{p} R
$$

for some $d>K_{1}$. We may assume that $\operatorname{cap}_{p} R$ is arbitrarily large because for any positive integer $m$ we may define

$$
R_{j}=\left\{x: r_{j-1}<\left|x-x_{0}\right|<r_{j}\right\}, \quad j=1, \ldots, m,
$$

where

$$
\begin{aligned}
r_{j} & =\left[a^{m-j} b^{j}\right]^{1 / m}, & & p=n, \\
& =\left[\left((m-j) a^{q}+j b^{q}\right) / m\right]^{1 / q}, & & p \neq n,
\end{aligned}
$$

and $q=(p-n) /(p-1)$. Then $\operatorname{cap}_{p} R_{j}=m^{p-1} \operatorname{cap}_{p} R$ and

$$
\sum_{j=1}^{m}\left(\operatorname{cap}_{p} R_{j}\right)^{1 /(1-p)}=\left(\operatorname{cap}_{p} R\right)^{1 /(1-p)}
$$

which together with the $p$-capacity version of [2, Lemma 2] gives

$$
d^{p} \operatorname{cap}_{p} R \leqq\left\{\sum_{j=1}^{m}\left(\operatorname{cap}_{p} T\left(R_{j}\right)\right)^{1 /(1-p)}\right\}^{1-p}
$$

An easy calculation shows that for some $1 \leqq k \leqq m$ we must have

$$
\operatorname{cap}_{p} T\left(R_{k}\right) \geqq m^{p-1} d^{p} \operatorname{cap}_{p} R=d^{p} \operatorname{cap}_{p} R_{k},
$$

and $R_{k}$ satisfies both (4.2) and $\operatorname{cap}_{p} R_{k}=m^{p-1} \operatorname{cap}_{p} R$. Thus we could use $R_{k}$ instead of $R$.

Now choose some $c$, with $K_{1}<c<d$, and assume

$$
\operatorname{cap}_{p} R>c^{p}(d-c)^{-p} \text {. }
$$

Let $v$ be the extremal function for $\operatorname{cap}_{p} R$ and set $u=v \circ T^{-1}$. Then by hypothesis $D_{p}[u] \geqq \operatorname{cap}_{p} T(R)$, and it follows from (4.1) and (4.2) that

$$
\begin{aligned}
K_{1}\|v\| & \geqq D_{p}[u]^{1 / p} \geqq\left(\operatorname{cap}_{p} T(R)\right)^{1 / p} \geqq d\left(\operatorname{cap}_{p} R\right)^{1 / p} \\
& =(d-c)\left(\operatorname{cap}_{p} R\right)^{1 / p}+c D_{p}[v]^{1 / p}>c\left(1+D_{p}[v]^{1 / p}\right)=c\|v\| .
\end{aligned}
$$

But this gives $c<K_{1}$, contradicting the choice of $c>K_{1}$ and proving the lemma. 
THEOREM 4.2. Every homeomorphism $T$ of $G$ onto $G^{\prime}$ for which $\varphi_{T}$ is an algebra isomorphism of $M_{p}\left(G^{\prime}\right)$ onto $M_{p}(G)$ is a $Q_{p}$-mapping.

Proof. Let $R$ be a spherical ring, $\bar{R} \subset G$, and let $v$ be the extremal function for $\operatorname{cap}_{p} R$. Then $\varphi_{T}^{-1}(v)=v \circ T^{-1}=u \in M_{p}\left(G^{\prime}\right)$, and $u$ is clearly admissible for $T(R)$. In $\S 6$ we apply some Banach algebra results (Lemma 6.1 ) to prove that $\varphi_{T}^{-1}$ is a bounded operator. Setting $K_{1}=\left\|\varphi_{T}^{-1}\right\|$ satisfies (4.1) and by Lemma 4.1 we have $T \in Q_{p}(K)$ for some $K \leqq K_{1}^{p}$. Applying the same argument to $T$ completes the proof.

Theorems 3.2 and 4.2 give the following characterization of a $Q_{p}$-mapping.

CoROllary 4.3. Let $T$ be a homeomorphism of $G$ onto $G^{\prime}$. Then $T$ is a $Q_{p}$-mapping if and only if $\varphi_{T}$ is an algebra isomorphism of $M_{p}\left(G^{\prime}\right)$ onto $M_{p}(G)$.

Another corollary follows from Theorem 4.2 and Remark 3.1.

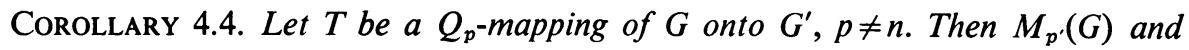
$M_{p^{\prime}}\left(G^{\prime}\right)$ are algebra isomorphic for every $p^{\prime}$.

COROLlaRY 4.5. $\varphi_{T}$ is an isometry if and only if $T, T^{-1} \in Q_{p}(1)$.

Proof. If $T, T^{-1} \in Q_{p}(1)$ then it follows that the constant $K_{2}=1$ in (3.5). For $p=n$ this is well known and for $p \neq n$ it follows from Remark 3.1 and the fact that $K_{0}=K^{2 n / p}$ in the proof of [5, Theorem 1]. Then (3.5) applied to $T$ and $T^{-1}$ implies that $D_{p}[f]=D_{p}\left[\varphi_{T}(f)\right], f \in M_{p}\left(G^{\prime}\right)$. But $\|f\|_{\infty}=\left\|\varphi_{T}(f)\right\|_{\infty}$ trivially, proving the isometry.

Conversely, if $\varphi_{T}$ is an isometry then (4.1) holds with $K_{1}=1$ and Lemma 4.1 implies $T \in Q_{p}(1)$. The same is true for $T^{-1}$, concluding the proof.

5. Sobolev spaces. With certain geometrical restrictions on the domains $G$ and $G^{\prime}$ the techniques of the previous section may be used to characterize those homeomorphisms whose composition maps the Sobolev spaces $H_{p}^{1}(G)$ and $H_{p}^{1}\left(G^{\prime}\right)$ onto each other.

Let $G_{1}$ be a convex subdomain of $G . G$ is star-shaped with respect to $G_{1}$ if $G$ contains every cone whose vertex is in $G$ and whose generators terminate on $G_{1}$, i.e., if $G$ is star-shaped with respect to every point of $G_{1}$. We first give a Sobolev imbedding lemma.

LEMMA 5.1. Let $G$ be a domain which is star-shaped with respect to some convex subdomain and which satisfies $m_{n}(G)<\infty$. Then there exists a constant $M$, depending only on $p$ and $G$, such that every $v \in H_{p}^{1}(G)$ satisfies

$$
\|v\|_{p} \leqq M\left(D_{p}[v]^{1 / p}+m_{n}(G)^{-1}\|v\|_{1}\right) .
$$

Proof. The cases $1<p \leqq n$ and $p>n$ are just special cases of Theorems 2 and 1 , respectively, of [18, pp. 56, 57]. Cf. also [7, pp. 369-380].

REMARK 5.2. Actually (5.1) holds for more general domains than those considered in Lemma 5.1. For example [7, Remark 4 and Theorem 2, p. 376], if 
$G=G_{1} \cup G_{2}$, where (5.1) holds for $G_{1}$ and $G_{2}$, and if $m_{n}\left(G_{1} \cap G_{2}\right)>0$, then (5.1) holds for $G$. See [10, Theorem 3.2.1 and pp. 72-74] for a still more general class of domains for which (5.1) holds.

THEOREM 5.3. Let $G$ and $G^{\prime}$ be domains of finite measure for which (5.1) holds, and let $T$ be a homeomorphism of $G$ onto $G^{\prime}$. Then $T$ is a $Q_{p}$-mapping if and only if $\varphi_{T}$ maps $H_{p}^{1}\left(G^{\prime}\right)$ onto $H_{p}^{1}(G)$.

Proof. If $T$ is a $Q_{p}$-mapping we use Ziemer's theorem [20] to prove that composition with $T$ preserves the $H_{p}^{1}$ classes. In case $p \neq n$ we conclude from [5, Lemma 7] and Remark 3.1 that $T$ is bi-measurable. (3.1) implies that $\left\|d T^{-1}\right\| \leqq M$, hence that $T^{-1}$ is of class $H_{p^{\prime}}^{1}$ on $G$, where $p^{\prime}=p(n-1) /(p-1)$. Then for any $1<p<\infty$ it follows from [20, Theorem 1.1], as in the proof of (3.5) for $p=n$, that $D_{p}[v] \leqq$ $K_{2} D_{p}[u]<\infty$ and $v \in H_{1}^{1}(G)$ whenever $v=u \circ T, u \in H_{p}^{1}\left(G^{\prime}\right)$. Thus $\|v\|_{1}<\infty$ and $D_{p}[v]<\infty$, and (5.1) implies $\|v\|_{p}<\infty$. Finally this means $v \in H_{p}^{1}(G)$ and $\varphi_{T}\left(H_{p}^{1}\left(G^{\prime}\right)\right)$ $\subset H_{p}^{1}(G)$. The opposite inclusion follows similarly, proving half of the theorem.

Conversely, if $\varphi_{T}$ maps $H_{p}^{1}\left(G^{\prime}\right)$ onto $H_{p}^{1}(G)$, then by Lemma 1.1 its restriction is actually an isomorphism between the Royden $p$-algebras, and the other half follows from Theorem 4.2.

REMARK 5.4. The boundedness of the partial derivatives of both $T$ and $T^{-1}$ is known to be sufficient for $T$ to preserve the $H_{p}^{1}$ classes [10, Theorems 3.1.5 and 3.1.6]. Theorem 5.3 shows that this condition is also necessary for $p \neq n$. It is not necessary for $p=n$, since the partial derivatives of a quasiconformal mapping need not be bounded.

6. The Royden $p$-compactification. We recall first some well-known facts about Banach algebras. Let $A$ be a normed algebra of continuous functions defined on $G$ which contains the constant functions. Suppose that $A$ is regular, i.e., that for every closed set $W \subset G$ and every $x \in G-W$ there exists some $f \in A$ such that $f=0$ on $W$ and $f(x) \neq 0$.

Denote by $G^{*}=G_{A}^{*}$ the collection of all nonzero bounded complex linear functionals $\chi$ on $A$ which satisfy

$$
\chi(f g)=\chi(f) \chi(g) \text { and } \chi(\bar{f})=\overline{\chi(f)}, \quad f, g \in A .
$$

Then $\|\chi\|=1$ for every $\chi \in G^{*}$, and $G^{*}$ is contained in the unit sphere of the dual space of $A$, inheriting the relative weak* topology generated by $A$. That is, $\chi_{\alpha} \rightarrow \chi$ in $G^{*}$ if and only if

$$
\lim _{\alpha}\left|\chi_{\alpha}(f)-\chi(f)\right|=0, \quad f \in A .
$$

In this topology $G^{*}$ is closed, and hence is a compact Hausdorff space by Alaoglu's theorem [16, p. 202]. For each $x \in G$ define

$$
\hat{x}(f)=f(x), \quad f \in A .
$$


Then since $A$ is regular, $x \rightarrow \hat{x}$ is a homeomorphism of $G$ onto a subset $\hat{G}=\hat{G}_{A}$ of $G^{*}$.

Suppose in addition that $A$ is selfadjoint and inverse-closed, i.e., that $f \in A$ implies $\bar{f} \in A$ and that $f \in A$ and $\inf _{G}|f|>0$ imply $1 / f=f^{-1} \in A$, respectively. For each $f \in A$ define

$$
\hat{f}(\chi)=\chi(f), \quad \chi \in G^{*} .
$$

Then $f \rightarrow \hat{f}$ is a homomorphism of $A$ onto a subset $\hat{A}$ of $C\left(G^{*}\right)$, which by the Stone-Weierstrass theorem is dense. It follows [12, p. 163] that $\hat{G}$ is dense in $G^{*}$ and that

$$
G^{*}-\hat{G}=\left\{\chi \in G^{*}: \chi(f)=0, f \in A_{0}\right\},
$$

where $A_{0}$ denotes those functions in $A$ with compact support in $G$. Since $G^{*}-\hat{G}$ is thus the intersection of a family of zero sets of continuous functions, $\hat{G}$ is open in $G^{*}$ and $\Delta=\Delta_{A}=G^{*}-\hat{G}$ is called the $A$-ideal boundary of $G$. For every $x \in G$, $\hat{f}(\hat{x})=\hat{x}(f)=f(x)$, and by identifying $G$ with its homeomorphic image $\hat{G}$ we may consider $\hat{f}$ to be a continuous extension of $f$ to $G^{*}$. Then $G^{*}=G_{A}^{*}$ is the $A$ compactification of $G$ [1, Chapter 9] (which is unique up to a homeomorphism which leaves $G$ fixed).

Note that $M_{p}(G)$ is regular since it contains $C_{0}^{\infty}(G)$. That $M_{p}(G)$ is selfadjoint is trivial, and it is easy to verify that it is inverse-closed. Thus we may apply the above theory to the Royden $p$-algebra, in which case $\Delta$ and $G^{*}$ are called the Royden $p$-ideal boundary and the Royden p-compactification, respectively, of $G$.

Since $M_{p}(G)$ separates points, it is semisimple, i.e., $f \rightarrow \hat{f}$ is one-to-one. Thus we have the following result $[9$, p. 76$]$ which has already been used in the proof of Theorem 4.2.

LEMMA 6.1. Let $\psi$ be an algebra homomorphism from a commutative Banach algebra onto $M_{p}(G)$. Then $\psi$ is a bounded linear operator.

Lemma 6.2. For $1<p \leqq n$, no point of the Royden p-ideal boundary $\Delta$ has a countable neighborhood basis.

Proof. Assuming the contrary as in [13, p. 558], let $\left\{U_{j}\right\}$ be a countable neighborhood basis for the topology at $\chi \in \Delta$ and let $\hat{V}_{j}=U_{j} \cap \hat{G}$. Then $\left\{V_{j}\right\}$ is a sequence of nonempty open subsets of $G$, and we may assume that $\bar{V}_{j+1} \subset V_{j}, j=1,2, \ldots$ For each $j, V_{j}-\bar{V}_{j+1}$ contains some ball $B\left(x_{j}, b_{j}\right)$. Define $R_{j}=B\left(x_{j}, b_{j}\right)-\mathrm{Cl}\left(B\left(x_{j}, a_{j}\right)\right)$, where

$$
\begin{aligned}
a_{j} & =b_{j} \exp -\left(2^{j} \omega_{n}\right)^{1 /(n-1)}, & & p=n, \\
& =b_{j}\left[1-q b_{j}^{-q}\left(2^{j} \omega_{n}\right)^{1 /(p-1)}\right]^{1 / q}, & & p<n ;
\end{aligned}
$$

then $\operatorname{cap}_{p} R_{j}=2^{-j}, j=1,2, \ldots$

Denote the extremal function for $\operatorname{cap}_{p} R_{j}$ by $v_{j}$ and set

$$
w_{k}(x)=\sum_{j=1}^{k} v_{j}(x) \text { and } w(x)=\sum_{j=1}^{\infty} v_{j}(x) .
$$


Since we may choose $v_{j}=0$ on $G-B\left(x_{j}, b_{j}\right)$ and since $\left\{B\left(x_{j}, b_{j}\right)\right\}$ are all disjoint, $\left\{w_{k}\right\}$ converges uniformly on compact subsets of $G$ to $w$, and $0 \leqq w \leqq 1$. Also $D_{p}\left[w_{k}-w\right]=2^{-k} \rightarrow 0$, and $w_{k} \rightarrow w$ in the $\mathrm{BD}_{p}$-topology. Then $w \in M_{p}(G)$ by Corollary 3.2, and $w$ has a continuous extension to $G^{*}$. Choosing $y_{j} \in S\left(x_{j}, b_{j}\right)$ gives $\hat{y}_{j} \rightarrow \chi$ with $\hat{w}\left(\hat{y}_{j}\right)=0$. But $\hat{x}_{j} \rightarrow \chi$ also and $\hat{w}\left(\hat{x}_{j}\right)=1$, contradicting the continuity of $\hat{w}$ and proving the lemma.

7. Induced homeomorphisms. Let $\psi$ be an algebra isomorphism of $M_{p}\left(G^{\prime}\right)$ onto $M_{p}(G)$. Then the adjoint mapping $T^{*}$, defined by

$$
T^{*}(\chi)=\chi \circ \psi, \quad \chi \in G^{*},
$$

is a homeomorphism of $G^{*}$ onto a closed subset of $G^{*}\left[9\right.$, p. 76]. Since $\psi^{-1}$ satisfies the same conditions it is clear that $T^{*}\left(G^{*}\right)=G^{*}$.

Suppose that $T^{*}(\hat{G})=\hat{G}^{\prime}$. Then composition with $x \rightarrow \hat{x}$ and its inverse would induce a homeomorphism $T$ of $G$ onto $G^{\prime}$ satisfying

$$
T(x)=y, \quad \text { where } \hat{y}=T^{*}(\hat{x}) .
$$

Note that the isomorphism $\psi$ would be given by composition with $T$, for let $f \in M_{p}\left(G^{\prime}\right)$. Then for any $x \in G$

$$
\psi(f)(x)=\hat{x} \circ \psi(f)=T^{*}(\hat{x})(f)=\hat{y}(f)=f \circ T(x),
$$

where $y=T(x)$.

THEOREM 7.1. For $1<p \leqq n$, every isomorphism $\psi$ of $M_{p}\left(G^{\prime}\right)$ onto $M_{p}(G)$ induces a $Q_{p}$-mapping $T$ of $G$ onto $G^{\prime}$ such that $\psi=\varphi_{T}$.

Proof. For $1<p \leqq n$, Lemma 6.2 implies that $T^{*}(\hat{G}) \subset \hat{G}^{\prime}$ and that $T^{*-1}\left(\hat{G}^{\prime}\right) \subset \hat{G}$. Thus the above argument holds, and (7.2) gives $\psi=\varphi_{T}$. Theorem 4.2 concludes the proof.

COROLlaRY 7.2. For $1<p \leqq n$ there is a one-to-one correspondence between $Q_{p^{-}}$ mappings of $G$ onto $G^{\prime}$ and algebra isomorphisms of $M_{p}(G)$ onto $M_{p}\left(G^{\prime}\right)$ given by $T \rightarrow \varphi_{T^{-1}}$.

COROLlaRY 7.3. $G$ and $G^{\prime}$ are quasiconformally equivalent if and only if $M_{p}(G)$ and $M_{p}\left(G^{\prime}\right)$ are algebra isomorphic for some $p$ satisfying $1<p \leqq n$.

REMARK 7.4. Using $[6, \S 10]$ we may now observe that $M_{3}(G)$ and $M_{3}\left(B^{3}\right)$ are not isomorphic if the boundary of $G$ has an inward directed spire or an outward directed ridge.

8. The Royden $p$-ideal boundary. We conclude with some remarks about the behavior of $\Delta$ under $Q_{p}$-mappings defined on $G$. The Royden p-harmonic boundary $\Gamma$ is defined by

$$
\Gamma=\left\{\chi \in G^{*}: \chi(f)=0, f \in M_{\Delta}(G)\right\}
$$


where $M_{\Delta}(G)$ denotes the closure in the $\mathrm{BD}_{p}$-topology of the functions in $M_{p}(G)$ with compact support in $G$. It follows from Corollary 2.2 and (6.1) that $\Gamma$ is a closed subset of $\Delta$.

THEOREM 8.1. Every $Q_{p}$-mapping $T$ of $G$ onto $G^{\prime}$ induces a homeomorphism of $\Delta$ onto $\Delta^{\prime}$ whose restriction to $\Gamma$ is a homeomorphism onto $\Gamma^{\prime}$.

Proof. By Theorem 3.2, $T$ induces an isomorphism $\varphi_{T}$ of $M_{p}\left(G^{\prime}\right)$ onto $M_{p}(G)$ which in turn induces a homeomorphism $T^{*}$ of $G^{*}$ onto $G^{*}$ defined by

$$
T^{*}(\chi)=\chi \circ \varphi_{T}, \quad \chi \in G^{*} .
$$

But for $x \in G$

$$
\begin{aligned}
T^{*}(\hat{x})(f) & =\hat{x} \circ \varphi_{T}(f) \\
& =\hat{x}(f \circ T)=f(y)=\hat{y}(f)
\end{aligned}
$$

for all $f \in M_{p}\left(G^{\prime}\right)$, where $y=T(x)$. Thus $T^{*}(\hat{G}) \subset \hat{G}^{\prime}$, and the opposite inclusion implies that the restriction of $T^{*}$ to $\Delta$ is the desired homeomorphism.

Now let $\chi \in \Gamma$ and let $\left\{f_{n}\right\}$ be a sequence of functions in $M_{p}(G)$ with compact support which converges in the $\mathrm{BD}_{p}$-topology to $f$. We show that $T^{*}(\chi)(f)=0$. Denote $g_{n}=f_{n} \circ T$ and $g=f \circ T$. Then (3.5) implies that $D_{p}\left[g_{n}-g\right] \rightarrow 0$, and $\left\{g_{n}\right\}$ converges to $g$ in the $\mathrm{BD}_{p}$-topology. But each $g_{n}$ has compact support and

$$
T^{*}(\chi)(f)=\chi \circ \varphi_{T}(f)=\chi(g)=0 .
$$

Thus $T^{*}(\Gamma) \subset \Gamma^{\prime}$, and the opposite inclusion completes the proof.

Added in proof. H. M. Reimann has recently made me aware of a simple example which shows that Theorem 7.1 is false for $p>n$.

\section{BIBLIOGRAPHY}

1. C. Constantinescu and A. Cornea, Ideale Ränder Riemannscher Flächen, Springer-Verlag, Berlin, 1963. MR 28 \#3151.

2. F. W. Gehring, Symmetrization of rings in space, Trans. Amer. Math. Soc. 101 (1961), 499-519. MR 24 \#A2677.

3. __ Rings and quasiconformal mappings in space, Trans. Amer. Math. Soc. 103 (1962), 353-393. MR 25 \#3166.

4. - Extremal length definitions for the conformal capacity of rings in space, Michigan Math. J. 9 (1962), 137-150. MR 25 \#4098.

5. - Lipschitz mappings and the p-capacity of rings in $n$-space, Proc. Sympos. Pure Math., vol. 20, Amer. Math. Soc., Providence, R. I., 1971.

6. F. W. Gehring and J. Väisälä, The coefficients of quasiconformality of domains in space, Acta Math. 114 (1965), 1-70. MR 31 \#4905.

7. L. V. Kantorovič and G. P. Akilov, Functional analysis in normed spaces, Fizmatgiz, Moscow, 1959; English transl., Internat. Series of Monographs in Pure and Appl. Math., vol. 46, Macmillan, New York, 1964. MR 22 \#9837; MR 35 \#4699.

8. C. Loewner, On the conformal capacity in space, J. Math. Mech. 8(1959), 411-414. MR 21 \#3538. 
9. L. H. Loomis, An introduction to abstract harmonic analysis, Van Nostrand, Princeton, N. J., 1953. MR 14, 883.

10. C. B. Morrey, Jr., Multiple integrals in the calculus of variations, Springer-Verlag, New York, 1966. MR 34 \#2380.

11. G. D. Mostow, Quasi-conformal mappings in n-space and the rigidity of hyperbolic space forms, Inst. Hautes Études Sci. Publ. Math. No. 34 (1968), 53-104. MR 38 \#4679.

12. M. Nakai, Algebraic criterion on quasiconformal equivalence of Riemann surfaces, Nagoya Math. J. 16 (1960), 157-184. MR 22 \#1669.

13. - Some topological properties on Royden's compactification of a Riemann surface, Proc. Japan. Acad. 36 (1960), 555-559. MR 23 \#A102.

14. H. M. Reimann, Uber harmonische Kapazität und quasikonforme Abbildungen im Raum, Comment. Math. Helv. 44 (1969), 284-307. MR 40 \#5856.

15. H. Royden, On the ideal boundary of a Riemann surface, Contributions to the Theory of Riemann Surfaces, Ann. of Math. Studies, no. 30, Princeton Univ. Press, Princeton, N. J. 1953. MR 15, 25.

16. ——, Real analysis, 2nd ed., Macmillan, New York, 1968.

17. S. Saks, Theory of the integral, 2nd rev. ed., Dover, New York, 1964. MR 29 \#4850.

18. S. Sobolev, Applications of functional analysis in mathematical physics, Izdat. Leningrad. Gos. Univ., Leningrad, 1950; English transl., Transl. Math. Monographs, vol. 7, Amer. Math. Soc., Providence, R. I., 1963. MR 14, 565; MR 29 \#2624.

19. J. Väisälä, On quasiconformal mappings in space, Ann. Acad. Sci. Fenn. Ser. A. I. No. 298 (1961), 1-36. MR 25 \#4100a.

20. W. Ziemer, Change of variables for absolutely continuous functions, Duke Math. J. 36 (1969), 171-178. MR 38 \#6006.

City University of New York, Graduate Center, NEW YorK, New YorK 10036

UNIVERSITY OF UTAH,

Salt Lake City, Utah 84112 